children with intracranial germ cell tumors. J Pediatr 2013 Nov;163(5):1448-53). (Reprints: Shannon M MacDonald MD, Dept. of Radiation Oncology, MGH, 55 Fruit St, Yawkey 112, Boston, MA 02114. E-mail: smacdonald@partners.org).

COMMENT. Bifocal germ cell tumors: synchronous tumors or metastases? Bifocal germ cell tumors in the suprasellar and pineal regions are reported in 23 (12.8\%) of 181 patients with intracranial GCTs treated at Seoul National University Children's Hospital, Korea (Phi JH, Kim SK, Lee J, et al. J Neurosurg Pediatr 2013 Feb;11(2):107-14). Eleven patients (47.8\%) presenting with bifocal GCTs exhibited tumor seeding, compatible with bifocal lesions. Patients with bifocal germinomas show significantly shorter survival than those with germinomas from a single site. Bifocal GCTs may result from the metastatic spread of suprasellar or pineal GCTs and are a sign of disseminated disease and poor prognosis.

\title{
NEUROMUSCULAR DISORDERS
}

\section{SPASMODIC MUSCLE CRAMPS AND WILSON DISEASE}

Investigators at Ann \& Robert H. Lurie Children's Hospital of Chicago report a case of Wilson disease (WD) in a 10-year-old-boy presenting with 3 months of increasingly severe spasmodic muscle cramps and weakness in lower extremities, upper extremities, and cramps in face and chest. Calf palpation was tender, and hyperpigmented flat lesions were present over ankles, knees, and elbows. Eye exam showed KayserFleischer rings and sunflower cataracts. Creatine kinase, aspartate aminotransferase, and alanine aminotransferase were elevated, hemoglobin was low, and urinalysis revealed myoglobinuria. MRI of muscle and muscle biopsy were negative, serum ceruloplasmin was low, $24 \mathrm{~h}$ urine copper was elevated, and liver biopsy showed fibrosis and positive staining for copper. Rhabdomyolysis developed after the operation, attributed to the use of succinylcholine. Brain MRI showed symmetric changes in the basal ganglia. Following trientene chelation therapy for WD, symptoms and laboratory abnormalities resolved. (Rosen JM, Kuntz N, Melin-Aldana H, Bass LM. Spasmodic muscle cramps and weakness as presenting symptoms in Wilson disease. Pediatrics 2013 Oct;132(4):e1039-42). (Response: John M Rosen MD, Children's Mercy Hospitals and Clinics, 2401 Gillham Road, Kansas City, MO 64108. E-mail: jmrosen@cmh.edu).

COMMENT. The clinical presentation of WD or hepatolenticular degeneration is variable and a number of different syndromes are recognized in addition to the classical syndrome described by Wilson in 1912. The classical syndrome, in children of 10 to 15 years, presents with bulbar symptoms. Rigidity of skeletal musculature follows, and an extrapyramidal type of hypertonus resembling that of the Parkinsonian syndrome is associated with a constant tremor. Tendon reflexes are usually normal. Any stimulus provokes spasms, and a diagnosis of tetanus may be entertained. Myotonia is also described. (Ford FR. Diseases of the Nervous System. In Infancy, Childhood and Adolescence. $4^{\text {th }}$ ed. Springfield, IL: Charles C Thomas; 1960. p. 756-62.). The Rosen, Kuntz et al case-report describes a WD syndrome with muscle cramps. 